\title{
Mediterranean diet and upper aerodigestive tract cancer: the Greek segment of the Alcohol-Related Cancers and Genetic Susceptibility in Europe study
}

\author{
Evangelia Samoli ${ }^{1}$, Areti Lagiou ${ }^{2}$, Elias Nikolopoulos ${ }^{3}$, Georgios Lagogiannis ${ }^{4}$, Anastasia Barbouni ${ }^{5}$, \\ Dimitrios Lefantzis ${ }^{3}$, Dimitrios Trichopoulos ${ }^{6,7}$, Paul Brennan ${ }^{8}$ and Pagona Lagiou ${ }^{1 *}$ \\ ${ }^{1}$ Department of Hygiene, Epidemiology and Medical Statistics, University of Athens Medical School, 75 Mikras Asias Street, \\ Goudi, GR-115 27 Athens, Greece \\ ${ }^{2}$ Faculty of Health Professions, Technological Educational Institute, Athens, Greece \\ ${ }^{3}$ Otorhinolaryngology Clinic, Red Cross Hospital, Athens, Greece \\ ${ }^{4}$ Maxillofacial Surgery Clinic, Agios Savas Hospital, Athens, Greece \\ ${ }^{5}$ Department of Public and Administrative Health, National School of Public Health, Athens, Greece \\ ${ }^{6}$ Department of Epidemiology, Harvard School of Public Health, Boston, MA, USA \\ ${ }^{7}$ Bureau of Epidemiologic Research, Academy of Athens, Athens, Greece \\ ${ }^{8}$ International Agency for Research on Cancer - IARC, Lyon, France
}

(Received 11 February 2010 - Revised 13 April 2010 - Accepted 26 April 2010 - First published online 26 May 2010)

Several dietary factors have been associated with the occurrence of cancers of the oral cavity and oropharynx, larynx and oesophagus, collectively called upper aerodigestive tract (UADT) cancers, but the evidence is considered as inconclusive. We hypothesised that the traditional Mediterranean dietary pattern may be more strongly inversely associated with UADT cancer risk than individual dietary components, and may explain the unexpectedly low incidence of these cancers in Greece. In the context of the European alcohol-related cancers and genetic susceptibility in Europe project, we have conducted a hospital-based case-control study in Athens, Greece, comparing 239 incident UADT cases and 194 hospital controls with admission diagnoses unrelated to tobacco, alcohol or diet. Adherence to Mediterranean diet was assessed through a widely used score, which ranges from 0 (minimal adherence) to 9 (maximal adherence) and increases with high consumption of plant foods and olive oil and low consumption of meat, dairy products and saturated lipids. Stricter adherence to the Mediterranean diet was associated with a substantial and significant decrease in UADT cancer risk (30\% for a two-unit increase in score), whereas after mutual adjustment, no individual dietary component of this diet was significantly associated with this risk. Adherence to the traditional Mediterranean diet is associated with reduced risk of UADT cancers, and may explain the lower incidence of UADT cancers in Greece, in spite of the smoking and drinking habits of this population.

Mediterranean diet: Upper aerodigestive tract: Cancers

Cancers of the oral cavity, oropharynx, larynx and oesophagus are frequently studied as a group of cancers of the upper aerodigestive tract (UADT) ${ }^{(1)}$, because they are believed to share, even in terms of quantitative importance, exogenous aetiological factors, notably tobacco ${ }^{(2)}$, alcohol ${ }^{(3,4)}$ and $\operatorname{diet}^{(4,5)}$, as well as genetic predisposition ${ }^{(6)}$. Worldwide, UADT cancers as a group are very common, with fatality generally exceeding $50 \%{ }^{(7)}$. In Greece, incidence of and mortality from UADT cancers are substantially lower than the corresponding indices in Western and Northern Europe and in the United States ${ }^{(7)}$. Because the prevalence of tobacco smoking is very high in Greece and consumption of alcoholic beverages is only slightly lower than that in the rest of Europe ${ }^{(8)}$, we hypothesised that the traditional Greek Mediterranean diet, which is still adhered to by large segments of the adult Greek population, may be responsible by conveying protection against
UADT cancers. To evaluate this hypothesis, we have used data from Greece collected in the context of the AlcoholRelated Cancers and Genetic Susceptibility in Europe (ARCAGE) study ${ }^{(1)}$.

\section{Materials and methods}

Study subjects

ARCAGE is a multicentre case-control study. The study was approved by the ethical review board of IARC, as well as by the respective local boards in the participating centres across Europe. All subjects provided written informed consent for their participation in the study. Details on the overall study design are given elsewhere ${ }^{(1)}$.

Abbreviations: ARCAGE, Alcohol-Related Cancers and Genetic Susceptibility in Europe; MDS, Mediterranean diet score; UADT, upper aerodigestive tract. 
The Greek component of the ARCAGE study was conducted from 2002 to 2005 in four major hospitals in Athens. Incident cases were identified in the hospitals, and were pathologically confirmed. Among eligible cases, approximately $4 \%$ were unable or not willing to participate. Eventually, a total of 239 cases were included in the study: 109 with cancer of the oral cavity or pharynx (excluding nasopharynx), 108 with cancer of the larynx and 22 with cancer of the oesophagus. In order to facilitate collection of blood samples, hospital controls were used and they were frequency matched to cases by sex and age (in 5-year groups). Only controls with a recently diagnosed nonmalignant disease were accepted, and admission diagnoses related to alcohol, tobacco or dietary practices were not eligible. The proportion of controls within a specific diagnostic group could not exceed $33 \%$ of the total ${ }^{(1)}$. Approximately $11 \%$ of the eligible controls were unable or not willing to participate. Eventually, a total of 194 controls were included in the study. Overall, the most common diagnoses among controls were gastrointestinal diseases (by protocol, appendicitis, anal fissure and fistula, perianal abscess, ischiorectal abscess and cholangitis) (14\%); diseases of the upper respiratory tract $(13 \%)$; skin, subcutaneous tissue and musculoskeletal conditions (12\%); trauma (12\%); circulatory system diseases $(10 \%)$; benign conditions of oral cavity, salivary gland and jaw $(8 \%)$; and genitourinary conditions $(7 \%)$.

Cases and controls underwent identical interviews in person during which a lifestyle questionnaire was completed with the help of a trained health professional. The questionnaire included information on socio-demographic variables, as well as detailed smoking and alcohol drinking histories. Height and weight at recruitment, as well as 2 years before the current hospitalisation, were also recorded, and BMI was calculated.

\section{Dietary intake and Mediterranean diet score}

In the Greek ARCAGE study, dietary habits were assessed using an extensive semi-quantitative FFQ, regularly used in case-control studies in Greece, and allowing for the calculation of total energy intake. Cases and controls were asked to indicate the average frequency of consumption per month, per week or per day of about 120 food items or beverage categories during a period of 1 year before the onset of the present disease. Using food composition tables that accommodate the particularities of the Greek diet ${ }^{(9)}$, frequencies of consumption were translated into quantities on the basis of typical portion sizes, and quantities were converted into average daily nutrient intakes. Consumption of alcoholic beverages, on account of its known association with UADT cancer risk, was recorded in detail using the analytical ARCAGE questionnaire on drinking habits, and ethanol content per type of drink was estimated according to the overall ARCAGE protocol ${ }^{(1)}$.

To evaluate adherence to the traditional Mediterranean diet, a score that was originally developed by Trichopoulou et al. ${ }^{(10)}$ and later revised to include fish intake ${ }^{(11)}$ was used. In brief, a value of 0 or 1 was assigned to each of nine components with the use of the sex-specific median as the cut-off. For beneficial components (vegetables, legumes, fruits and nuts, cereals and fish), persons whose consumption was below the median were assigned a value of 0 , and persons whose consumption was at or above the median were assigned a value of 1. For components presumed not to be beneficial (meat and dairy products), persons whose consumption was below the median were assigned a value of 1 , and persons whose consumption was at or above the median were assigned a value of 0 . To assess lipid intake, the ratio of monounsaturated to saturated lipids was used, and the value of 1 was assigned to those with a ratio equal to or above the sex-specific median. For ethanol, we assigned a value of 1 to men who consumed between 10 and $50 \mathrm{~g} / \mathrm{d}$ and to women who consumed between 5 and $25 \mathrm{~g} / \mathrm{d}$, based on average glasses of alcoholic beverages per day among current or former drinkers. The cut-off points for alcohol consumption in the context of the Mediterranean diet have been proposed in $1995^{(10)}$, and have been consistently used since ${ }^{(11)}$. Thus, the total Mediterranean diet score (MDS) ranged from 0 (minimal adherence to the traditional Mediterranean diet) to 9 (maximal adherence).

\section{Statistical analysis}

For the statistical analysis, all UADT cancer cases were grouped together, because they were considered to share common aetiological factors in tobacco smoking, consumption of alcoholic beverages and diet. Simple tabulations were used to examine the distribution of cases and controls by sex, years of schooling, tobacco smoking and alcohol drinking. For age, height and BMI 2 years before diagnosis, mean values with their standard errors were calculated. With respect to consumption of the components of the MDS, energy-adjusted (to $8368 \mathrm{~kJ} / \mathrm{d}$ for men and to $7113 \mathrm{~kJ} / \mathrm{d}$ for women) mean daily intakes by sex and case-control status were estimated.

The association of Mediterranean diet with UADT cancer risk was assessed by multiple logistic regression. We evaluated all nine components, considered as dichotomous, using the sex-specific medians as cut-offs (except for alcohol), at first alternatively and then simultaneously. Subsequently, we evaluated the association of the MDS with UADT cancer risk for two- and three-unit increments. Due to the established detrimental effect of ethanol intake in the occurrence of UADT $_{\text {cancers }}{ }^{(4)}$, we also evaluated the risk associated with MDS by excluding the ethanol component (thus reducing the ten-point score to a nine-point score), and estimated the OR associated with a two- and three-unit increment in the score minus ethanol. To preserve comparability, we multiplied the logarithm of the estimated OR by $9 / 10$ before exponentiating.

In all models, we controlled for age (in years, continuous), sex, BMI 2 years before diagnosis $\left(\mathrm{kg} / \mathrm{m}^{2}\right.$, ordered in quintiles), height (in $\mathrm{cm}$, continuous), educational level (categorical: primary as baseline, higher but not university-level education and university education) and smoking status (never smokers, former smokers and current smokers; for former and current smokers, further control was undertaken for pack-years as an ordered variable with $1=<20$, $2=20-39, \quad 3=40-59, \quad 4=60-79$ and $5=80+$ packyears). Pack-years among ex-smokers and current smokers were assessed as ordinal variables to avoid disproportional influence of a few individuals with extreme values. We also controlled for alcohol intake (moderate $v$. others) in all models except in the ones that focused on it as a distinct variable and the model evaluating the association of the 
overall MDS (which includes a component for alcohol) with UADT. In the design of the analytical strategy, we considered known, or suspected, risk factors as the possible confounding variables for the disease outcome. Although many of the potential confounders had minimal confounding influence, we consistently adjusted for them in the models to preserve comparability of the estimates. Statistical analysis was conducted using the statistical package for social sciences 16.0 statistical software (SPSS version 16; SPSS, Inc., Chicago, IL, USA).

\section{Results}

Table 1 presents basic demographic characteristics, as well as patterns of tobacco smoking and alcohol consumption, for 239 participants with UADT cancer and 194 control subjects. These data serve only descriptive purposes because the indicated variables are not mutually adjusted for. In comparison to control subjects, cancer patients were somewhat less educated, heavier smokers and more frequently drinkers. All these differences were accounted for in the statistical modelling of the nutritional data.

Table 2 allows an inspection of differences between cases and controls in the energy-adjusted daily intakes of components of the MDS by sex. These comparisons are not adjusted for demographic or lifestyle variables. There is evidence that intake of meat is associated with increased risk of UADT cancers, whereas consumption of vegetables or fruits is inversely associated with UADT cancer risk.

Table 3 presents mutually adjusted OR and 95\% CI for UADT cancers by anthropometric as well as non-dietary lifestyle characteristics. There appears to be no important association of UADT cancer risk with educational level or somatometric characteristics. As expected, smoking is a major risk factor for developing UADT cancers, and heavy (former or current) drinkers are at increased risk than nondrinkers or light drinkers.

Table 4 presents the OR and $95 \%$ CI for UADT cancers by components of the MDS, controlling for the anthropometric and lifestyle characteristics listed in Table 3. Results are given with and without mutual adjustment among the indicated nutritional variables. Higher consumption of vegetables $(\mathrm{OR}=0.62,95 \%$ CI $0.39,1.00)$ and legumes $(\mathrm{OR}=0.61$, $95 \%$ CI $0.39,0.95)$ was significantly associated with reduced UADT cancer risk, but after mutual adjustment among the nine components of the MDS, no association remained significant at the 0.05 level, although associations were generally in the predicted directions.

Table 5 presents the OR and $95 \%$ CI for UADT cancers per two- and three-unit increase in the MDS. It also provides information on how the association of MDS with UADT cancer risk changes, when ethanol intake is not included in the score, but it is controlled for separately in the models (due to the known association of alcohol intake with UADT cancers, any benefit from adherence to the Mediterranean diet would be expected to increase after exclusion of ethanol from the score). Stricter adherence to the Mediterranean diet is associated with a substantial and significant decrease in UADT cancer risk $(30 \%$ for a two-unit increase and $41 \%$ for a three-unit increase in MDS), which becomes more evident $(31 \%$ for a two-unit increase and $42 \%$ for a three-unit increase in MDS without ethanol) and statistically more significant after exclusion of ethanol intake from the score.

Table 1. Characteristics* of incident cases of upper aerodigestive tract cancers and hospital controls in the Greek segment of the Alcohol-Related Cancers and Genetic Susceptibility in Europe study

\begin{tabular}{|c|c|c|c|c|c|}
\hline & \multicolumn{2}{|c|}{ Cases (n 239) } & \multicolumn{2}{|c|}{ Controls ( $n$ 194) } & \multirow[b]{2}{*}{$P \dagger$} \\
\hline & Mean $/ n$ & $\mathrm{SE} / \%$ & Mean/n & $\mathrm{SE} / \%$ & \\
\hline Age (years) & $61 \cdot 3$ & $0 \cdot 8$ & $60 \cdot 6$ & 1.0 & 0.527 \\
\hline Sex & & & & & 0.101 \\
\hline Males & 192 & $80 \cdot 3$ & 143 & $73 \cdot 7$ & \\
\hline Females & 47 & $19 \cdot 7$ & 51 & $26 \cdot 3$ & \\
\hline Height (cm) & $169 \cdot 0$ & 0.6 & $169 \cdot 0$ & 0.6 & 0.676 \\
\hline BMI $\left(\mathrm{kg} / \mathrm{m}^{2}\right)$ & $26 \cdot 0$ & 0.3 & $26 \cdot 9$ & 0.3 & 0.045 \\
\hline Years of schooling & & & & & 0.022 \\
\hline$<6$ & 148 & 61.9 & 100 & 51.5 & \\
\hline$>6-12$ & 73 & 30.5 & 71 & $36 \cdot 6$ & \\
\hline$>12$ & 18 & 7.5 & 23 & 11.9 & \\
\hline Smoking status & & & & & $<0.001$ \\
\hline Never smokers & 26 & $10 \cdot 9$ & 60 & $30 \cdot 9$ & \\
\hline Former smokers & 49 & 20.5 & 59 & $30 \cdot 4$ & \\
\hline Current smokers & 164 & 68.5 & 75 & $38 \cdot 7$ & \\
\hline \multicolumn{6}{|l|}{ Pack-years of smoking } \\
\hline Among former smokers & $47 \cdot 0$ & 4.8 & 34.6 & $3 \cdot 7$ & 0.039 \\
\hline Among current smokers & $56 \cdot 8$ & $2 \cdot 7$ & 37.4 & 2.9 & $<0.001$ \\
\hline Alcohol drinking & & & & & 0.094 \\
\hline Never drinker & 26 & $10 \cdot 9$ & 35 & $18 \cdot 0$ & \\
\hline Former drinker & 20 & 8.4 & 11 & $5 \cdot 7$ & \\
\hline Current drinker & 193 & $80 \cdot 8$ & 148 & $76 \cdot 3$ & \\
\hline
\end{tabular}


Table 2. Energy-adjusted ${ }^{\star}$ mean daily intake of the nine components of the Mediterranean diet score by sex and upper aerodigestive tract cancer case-control status in the Greek segment of the Alcohol-Related Cancers and Genetic Susceptibility in Europe study

\begin{tabular}{|c|c|c|c|c|c|c|c|c|}
\hline & \multicolumn{4}{|c|}{ Males } & \multicolumn{4}{|c|}{ Females } \\
\hline & \multicolumn{2}{|c|}{ Cases } & \multicolumn{2}{|c|}{ Controls } & \multicolumn{2}{|c|}{ Cases } & \multicolumn{2}{|c|}{ Controls } \\
\hline Vegetables $(\mathrm{g} / \mathrm{d})$ & \multicolumn{2}{|c|}{$440 \cdot 8$} & \multicolumn{2}{|c|}{$505 \cdot 5$} & \multicolumn{2}{|c|}{$431 \cdot 0$} & \multicolumn{2}{|c|}{$503 \cdot 2$} \\
\hline Legumes $(\mathrm{g} / \mathrm{d})$ & \multicolumn{2}{|c|}{32.4} & \multicolumn{2}{|c|}{$32 \cdot 2$} & \multicolumn{2}{|c|}{23.7} & \multicolumn{2}{|c|}{$29 \cdot 1$} \\
\hline Fruits and nuts $(g / d)$ & \multicolumn{2}{|c|}{$432 \cdot 6$} & \multicolumn{2}{|c|}{483.4} & \multicolumn{2}{|c|}{$405 \cdot 2$} & \multicolumn{2}{|c|}{$499 \cdot 9$} \\
\hline Dairy products (g/d) & \multicolumn{2}{|c|}{$179 \cdot 9$} & \multicolumn{2}{|c|}{$188 \cdot 6$} & \multicolumn{2}{|c|}{244.9} & \multicolumn{2}{|c|}{$186 \cdot 0$} \\
\hline Cereals $(g / d)$ & \multirow{2}{*}{\multicolumn{2}{|c|}{$\begin{array}{l}194.4 \\
103.2\end{array}$}} & \multicolumn{2}{|c|}{205.5} & \multicolumn{2}{|c|}{$171 \cdot 1$} & \multicolumn{2}{|c|}{$179 \cdot 9$} \\
\hline Meat $(\mathrm{g} / \mathrm{d})$ & & & \multirow{2}{*}{\multicolumn{2}{|c|}{$\begin{array}{l}90 \cdot 8 \\
18 \cdot 4\end{array}$}} & \multicolumn{2}{|c|}{$99 \cdot 5$} & \multicolumn{2}{|c|}{$97 \cdot 7$} \\
\hline Fish $(g / d)$ & \multicolumn{2}{|c|}{$20 \cdot 0$} & & & & & & \\
\hline Ratio of monounsaturated to saturated lipids & \multicolumn{2}{|c|}{$2 \cdot 2$} & \multicolumn{2}{|c|}{$2 \cdot 3$} & \multicolumn{2}{|c|}{$2 \cdot 1$} & \multicolumn{2}{|c|}{$2 \cdot 3$} \\
\hline Ethanol intake $\dagger$ & $n$ & $\%$ & $n$ & $\%$ & $n$ & $\%$ & $n$ & $\%$ \\
\hline Non- or light drinkers & 64 & 33.3 & 73 & $51 \cdot 0$ & 39 & 83.0 & 44 & $86 \cdot 3$ \\
\hline Moderate drinkers & 90 & 46.9 & 56 & 39.2 & 6 & $12 \cdot 8$ & 7 & $13 \cdot 7$ \\
\hline Heavy drinkers & 38 & 19.8 & 14 & 9.8 & 2 & $4 \cdot 3$ & 0 & 0.0 \\
\hline
\end{tabular}

* To $8368 \mathrm{~kJ} / \mathrm{d}$ for men and to $7113 \mathrm{~kJ} / \mathrm{d}$ for women.

† For males, $<10,10-49.99$ and $50+\mathrm{g} / \mathrm{d}$. For females, $<5,5-24.99$ and $25+\mathrm{g} / \mathrm{d}$; classification was based on average glasses of alcoholic beverages/d among current or past drinkers.

\section{Discussion}

In a moderately sized case-control study of UADT cancers in the greater Athens area, we found that closer adherence to the traditional Mediterranean diet was strongly and significantly inversely associated with UADT cancer risk. In the present study, though the associations of each of the components of this diet with UADT cancer risk were generally in the expected direction, none reached statistical significance. We interpret the present finding as indicating that traditional Mediterranean diet is more important than its individual components in conveying protection against UADT cancers,

Table 3. Logistic regression-derived OR and $95 \% \mathrm{Cl}^{*}$ for upper aerodigestive tract cancers by specified variables in the Greek segment of the Alcohol-Related Cancers and Genetic Susceptibility in Europe study

(Odds ratios and $95 \%$ confidence intervals)

\begin{tabular}{|c|c|c|c|}
\hline & OR & $95 \% \mathrm{Cl}$ & $P$ \\
\hline $\mathrm{BMI}\left(\mathrm{kg} / \mathrm{m}^{2} ;\right.$ per quintile increase) & 0.91 & $0.78,1.07$ & 0.260 \\
\hline Height (per $5 \mathrm{~cm}$ ) & 1.05 & $0.89,1.24$ & 0.610 \\
\hline \multicolumn{4}{|l|}{ Years of schooling } \\
\hline$<6$ & & Baseline & \\
\hline$>6-12$ & $1 \cdot 19$ & $0.56,2.53$ & 0.655 \\
\hline$>12$ & 1.04 & $0.48,2.26$ & 0.930 \\
\hline \multicolumn{4}{|l|}{ Smoking status } \\
\hline Never smokers & & Baseline & \\
\hline Former smokers & 2.40 & $1.19,4.85$ & 0.015 \\
\hline Current smokers & $6 \cdot 33$ & $3 \cdot 26,12 \cdot 31$ & $<0.001$ \\
\hline \multicolumn{4}{|l|}{ Pack-years (per 1 pack-year) } \\
\hline Among former smokers & 1.39 & $1.00,1.92$ & 0.052 \\
\hline Among current smokers & 1.85 & $1.40,2.44$ & $<0.001$ \\
\hline \multicolumn{4}{|l|}{ Alcohol drinking $†$} \\
\hline Non- or light drinker & & Baseline & \\
\hline Moderate drinker & 1.24 & $0.76,2.03$ & 0.390 \\
\hline Heavy drinker & 2.08 & $0.96,4.51$ & 0.063 \\
\hline
\end{tabular}

* Adjusted for age and sex, as well as mutually among the indicated variables.

† For males, <10, 10-49.99 and $50+\mathrm{g} / \mathrm{d}$. For females, <5, 5-24.99 and $25+\mathrm{g} / \mathrm{d}$; classification was based on average glasses of alcoholic beverages/d among current or past drinkers. and that adherence to this diet could be largely responsible for the relatively low incidence of and mortality from these cancers in Greece.

The MDS is an a priori developed score ${ }^{(12)}$ relying on the salient characteristics of the traditional Mediterranean diet and the empirically derived evidence on the association of its components with overall mortality ${ }^{(10,11,13)}$. In this score, moderate consumption of alcohol, mostly in the form of wine during meals, is considered as conducive to health, mainly on account of the well-established U-shaped association of alcohol intake with cardiovascular mortality ${ }^{(14,15)}$, which is a common cause of death. For UADT cancer risk, however, the association with alcohol drinking is monotonic, which suggests that exclusion of the alcohol component from the MDS would strengthen the inverse association between the score and UADT cancer risk. This is indeed what happens (Table 5), although the change is marginal, probably because moderate - as contrasted to heavy - drinking only slightly increases UADT cancer risk in comparison to non-drinking (Table 4).

There are several reasons why the MDS is more strongly associated with UADT cancer risk than its individual components $^{(11,13,16)}$. It may be that chance, non-differential misclassification and residual confounding have more important consequences when a single food is evaluated rather than a multicomponent, unidimensional score, as their effects are more likely to disruptively operate on a single food group rather than simultaneously on several of them. Moreover, in an analysis focusing on individual components, effects are examined against the background of average risk associated with other nutritional components, whereas a dietary score can account for extremes of cumulative exposures in the absence of other major nutritional effects ${ }^{(16)}$.

There has been another and larger study examining the association of adherence to Mediterranean diet with cancers of the UADT ${ }^{(17)}$. The results were compatible with those of the present study. The Bosetti et al. study, however, relied on populations of northern Italy, where dietary practices are 
Table 4. Logistic regression-derived $\mathrm{OR}$ and $95 \% \mathrm{Cl}$ for upper aerodigestive tract cancers by components of the Mediterranean diet score in the Greek segment of the Alcohol-Related Cancers and Genetic Susceptibility in Europe study

(Odds ratios and $95 \%$ confidence intervals)

\begin{tabular}{|c|c|c|c|c|c|c|}
\hline & \multicolumn{3}{|c|}{ Unadjusted for each other* } & \multicolumn{3}{|c|}{ Mutually adjusted $\dagger$} \\
\hline & OR & $95 \% \mathrm{Cl}$ & $P$ & OR & $95 \% \mathrm{Cl}$ & $P$ \\
\hline Vegetables ( $\geq$ median $v$. $<$ median) & 0.62 & $0.39,1.00$ & 0.049 & 0.74 & $0.45,1.21$ & 0.225 \\
\hline Legumes $(\geq$ median $v .<$ median) & 0.61 & $0.39,0.95$ & 0.028 & 0.64 & $0.40,1.01$ & 0.055 \\
\hline Fruits and nuts ( $\geq$ median $v .<$ median) & 0.79 & $0.50,1.25$ & 0.318 & 0.84 & $0.52,1.35$ & 0.469 \\
\hline Dairy products ( $\geq$ median $v .<$ median) & 1.28 & $0.82,2.02$ & 0.280 & $1 \cdot 30$ & $0.80,2 \cdot 10$ & 0.297 \\
\hline Cereals ( $\geq$ median $v .<$ median) & 1.23 & $0.76,2 \cdot 00$ & 0.404 & $1 \cdot 18$ & $0.72,1.92$ & 0.518 \\
\hline Fish ( $\geq$ median $v .<$ median) & 0.69 & $0.44,1.07$ & 0.094 & 0.73 & $0.46,1.15$ & 0.168 \\
\hline Meat and meat products ( $\geq$ median $v$. $<$ median) & $1 \cdot 18$ & $0.75,1.88$ & 0.474 & $1 \cdot 13$ & $0.70,1.83$ & 0.608 \\
\hline Monounsaturated to saturated lipids ( $\geq$ median $v .<$ median) & 0.80 & $0.51,1.27$ & 0.348 & 0.99 & $0.61,1.61$ & 0.956 \\
\hline \multicolumn{7}{|l|}{ Alcohol drinkingł } \\
\hline Moderate drinkers $v$. all others & 1.07 & $0.67,1.70$ & 0.784 & 1.06 & $0.66,1.71$ & 0.810 \\
\hline Moderate $v$. non- and light drinkers & $1 \cdot 21$ & $0.74,1.98$ & 0.451 & 1.25 & $0.75,2.08$ & 0.393 \\
\hline Heavy $v$. non- and light drinkers & 1.76 & $0.80,3.90$ & $0 \cdot 162$ & 2.08 & $0.91,4.78$ & 0.084 \\
\hline
\end{tabular}

* Controlling for the variables listed in Table 4 as well as for energy intake.

$\dagger$ As in footnote $\left.{ }^{*}\right)$, but also mutually.

† For males, non-drinkers, $<10 \mathrm{~g} / \mathrm{d}$; moderate drinkers, $10-49.99 \mathrm{~g} / \mathrm{d}$; and heavy drinkers, $50+\mathrm{g} / \mathrm{d}$. For females, non-drinkers, $<5 \mathrm{~g} / \mathrm{d}$; moderate drinkers, $5-24.99 \mathrm{~g} / \mathrm{d}$; and heavy drinkers, $25+\mathrm{g} / \mathrm{d}$.

not typically Mediterranean. In any case, the convergence of the results of these two studies indicates that the inverse association between adherence to Mediterranean diet and UADT cancer risk is probably genuine and quite strong. Although there are several variations of the traditional diet in the olive oil-producing areas of the Mediterranean region, the MDS we have used captures the salient characteristics of this traditional diet and has been found to be robust to these variations $^{(18)}$.

The strengths of our investigation are the high participation rates among both cases and controls, the reliance on a strict protocol for case ascertainment and control selection, and the use of an extensive dietary questionnaire, shorter versions of which have been frequently employed in Greece in casecontrol studies with results in line with those of subsequent major cohort investigations ${ }^{(19,20)}$. Another strength of our investigation is also its undertaking in a population where large segments still adhere to the traditional Mediterranean diet. Weaknesses of our investigation include the modest study size and reliance on a hospital-based case-control design.

Table 5. Logistic regression-derived OR and $95 \% \mathrm{Cl}$ for upper aerodigestive tract cancers associated with two- or three-unit increase in the Mediterranean diet score* in the Greek segment of the Alcohol-Related Cancers and Genetic Susceptibility in Europe study

(Odds ratios and $95 \%$ confidence intervals)

\begin{tabular}{lccc}
\hline & OR & $95 \% \mathrm{Cl}$ & $P$ \\
\hline Overall Mediterranean diet score & & & 0.013 \\
Two-unit increase & 0.70 & $0.54,0.93$ & \\
Three-unit increase & 0.59 & $0.39,0.89$ & \\
Minus ethanol† & & & 0.006 \\
Two-unit increase & 0.69 & $0.53,0.90$ & \\
Three-unit increase & 0.58 & $0.39,0.85$ & \\
\hline
\end{tabular}

* Adjusted for age, sex, height, BMI, educational level, smoking status and packyears, and energy intake.

$\dagger$ Adjusted as in footnote $\left(^{*}\right)$, as well as for alcohol drinking (non- or light, moderate and heavy drinkers). Originally estimated logarithm of OR was multiplied by $9 / 10$, and then exponentiated to account for the proportionally larger increment in the minus ethanol score.
Hospital-based case-control studies are criticised for being susceptible to selection and information bias. However, control selection relied on the very strict ARCAGE protocol, which excluded admission diagnoses related to alcohol, smoking or diet, thus, limiting the potential for diagnosis-related selection bias. Moreover, choice of hospital controls maximised response rates and, thus, minimised participationrelated selection bias. Of note, the results concerning the relationship of individual components of the Mediterranean diet with UADT cancer risk were compatible with the collective evidence concerning the association of these factors with UADT cancer risk ${ }^{(4,21)}$.

In conclusion, we have found evidence that adherence to the traditional Mediterranean diet is associated with reduced risk of cancers of the UADT. The association is statistically significant and stronger than the associations of the individual components of this diet with UADT cancer risk. Closer adherence to the traditional Mediterranean diet could explain the lower incidence of UADT cancers in Greece, in spite of the smoking and drinking habits of this population.

\section{Acknowledgements}

The financial support for the present study was provided by the European Community (Fifth Framework Programme) grant no. QLK1-CT-2001-00182 and the University of Athens Medical School. None of the authors has any conflict of interest. E. S. was responsible for the statistical analysis. A. L. was responsible for the nutritional epidemiological analysis. E. N., G. L. and D. L. contributed to the data collection, and provided significant advice regarding clinical issues and the ascertainment of case diagnosis. A. B. contributed to the data processing and analysis. D. T. is a senior consultant epidemiologist. P. B. is the principal investigator of the overall ARCAGE project. P. L. is the principal investigator of the Greek ARCAGE study, and supervised the present study. P. L., E. S., A. L. and D. T. were responsible for the drafting 
of the manuscript with input from all authors. All authors have approved the submitted version of the manuscript.

\section{References}

1. Lagiou P, Georgila C, Minaki P, et al. (2009) Alcohol-related cancers and genetic susceptibility in Europe - the ARCAGE project: study samples and data collection. Eur J Cancer Prev 18, 76-84.

2. IARC (2004) Monographs on the Evaluation of Carcinogenic Risks to Human, vol. 83: Tobacco Smoke and Involuntary Smoking. Lyon, France: IARC Press.

3. IARC (2007) Monographs Working Group on Alcoholic Beverage Consumption and Ethyl Carbamate (Urethane), 6-13 February 2007. http://monographs.iarc.fr/ENG/Meetings/ vol96-summary.pdf

4. World Cancer Research Fund/American Institute for Cancer Research (2007) Food, Nutrition, Physical Activity, and the Prevention of Cancer: a Global Perspective. Washington, DC: AICR.

5. IARC (2003) Fruit and Vegetables. IARC Handbooks of Cancer Prevention, vol. 8, Lyon, France: IARC Press.

6. Canova C, Hashibe M, Simonato L, et al. (2009) Genetic associations of 115 polymorphisms with cancers of the upper aerodigestive tract across 10 European countries: the ARCAGE project. Cancer Res 69, 2956-2965.

7. Ferlay J, Parkin DM, Bray F, et al. (2004) Descriptive Epidemiology Group International Agency for Research on Cancer. Lyon, France: IARC Press.

8. WHO, Regional Office for Europe (2009) European Health for All Database (HFA-DB). Geneva: WHO.

9. Trichopoulou A \& Georga L (2004) Composition Tables of Foods and Greek Dishes, 3rd ed. Athens: Parisianou.
10. Trichopoulou A, Kouris-Blazos A, Wahlqvist ML, et al. (1995) Diet and overall survival in elderly people. BMJ 311, $1457-1460$

11. Trichopoulou A, Costacou T, Bamia C, et al. (2003) Adherence to a Mediterranean diet and survival in a Greek population. $N$ Engl J Med 348, 2599-2608.

12. Trichopoulos D \& Lagiou P (2001) Dietary patterns and mortality. Br J Nutr 85, 133-134.

13. Trichopoulou A, Bamia C \& Trichopoulos D (2009) Anatomy of health effects of Mediterranean diet: Greek EPIC prospective cohort study. BMJ 338, b2337.

14. Di Castelnuovo A, Costanzo S, Bagnardi V, et al. (2006) Alcohol dosing and total mortality in men and women. Arch Intern Med 166, 2437-2445.

15. Mukamal KJ \& Rimm EB (2008) Alcohol consumption: risks and benefits. Curr Atheroscler Rep 10, 536-543.

16. Jacques PF \& Tucker KL (2001) Are dietary patterns useful for understanding the role of diet in chronic disease? Am J Clin Nutr 73, 1-2.

17. Bosetti C, Gallus S, Trichopoulou A, et al. (2003) Influence of the Mediterranean diet on the risk of cancers of the upper aerodigestive tract. Cancer Epidemiol Biomarkers Prev 12, $1091-1094$.

18. Sofi F, Cesari F, Abbate R, et al. (2008) Adherence to Mediterranean diet and health status: meta-analysis. BMJ 337, a1344.

19. Manousos O, Day N, Trichopoulos D, et al. (1983) Diet and colorectal cancer: a case-control study in Greece. Int $J$ Cancer 32, 1-5.

20. Manousos O, Day N, Tzonou A, et al. (1985) Diet and other factors in the aetiology of diverticulosis: an epidemiological study in Greece. Gut 26, 544-549.

21. Lagiou P, Talamini R, Samoli E, et al. (2009) Diet and upperaerodigestive tract cancer in Europe: the ARCAGE study. Int J Cancer 124, 2671-2976. 